\title{
ANSIEDADE E DEPRESSÃO EM UMA AMOSTRA DE PACIENTES CLASSIFICADOS COMO PORTANDO FATORES PSICOLÓGICOS QUE AFETAM AS CONDIÇÕES MÉDICAS*
}

\author{
ANXIETY AND DEPRESSION IN A SAMPLE OF PATIENTS \\ CLASSIFIED AS CARRING PSYCHOLOGICAL FACTORS THAT \\ AFFECT MEDICAL CONDITIONS
}

Nicodemos Batista BORGES ${ }^{1}$

Gildo dos Santos ANGELOTTI

\begin{abstract}
RESUMO
Este estudo visou levantar fatores comportamentais, mais especificamente ansiedade e depressão, em uma amostra de pacientes classificados como apresentando Fatores Psicológicos que Afetam as Condições Médicas. Foram sujeitos do presente estudo: 30 pacientes que procuraram um Hospital Geral localizado na periferia da Zona Sul da cidade de São Paulo, de ambos os sexos e que se enquadravam na classificação acima. O instrumento utilizado foi a Escala Fatorial de Ajustamento Emocional/Neuroticismo (EFN), desenvolvida por Hutz e Nunes (2001). Como principais resultados encontraram-se: $36,67 \%$ dos sujeitos acima da média e 16,67\% muito acima da média, na sub-escala de ansiedade, e 43,33\% acima da média e $36,67 \%$ muito acima da média, na sub-escala de depressão.
\end{abstract}

Palavras-chave: depressão; ansiedade; fatores psicológicos que afetam condição médica, doença multifatorial.

\begin{abstract}
This paper aimed to characterize psychological factors as anxiety and depression in a sample of patients classified as carring psychological factors that affect medical conditions. It was subjects of the investigation:

\footnotetext{
(*) Agradecemos ao Estatístico e Professor Gilberto Mitsuo Ukita pelo auxílio no tratamento estatístico.

(1) Universidade de Santo Amaro - UNISA e Universidade de São Paulo - USP

Endereço para correspondência: E-mail: nicodemosborges@uol.com.br Rua Antonio Ambuba, 80 - casa 66 Horto do Ypê - São Paulo - SP CEP 05782-370 - Tel. (11) 5511-4925 / (11) 9386-4660

(2) Universidade de Santo Amaro - UNISA e Universidade Federal de São Paulo - UNIFESP
} 


\begin{abstract}
30 patients that sought a general hospital localized in the suburban area of São Paulo city south zone, of both sexes that was framed in classification above. It was utilized Factorial Scale of Emotional Adjustment/Neuroticism (EFN), developed by Hutz \& Nunes (2001). As principal results it has been found: $36,67 \%$ of the subjects above the average and $16,67 \%$ much above the average, in sub-scale of anxiety, and 43,33\% above the average and 36,67\% much above the average, in sub-scale of depression.
\end{abstract}

Keywords: depression; anxiety; psychological factors that affect medical condition, multifactorial disease.

\section{INTRODUÇÃO}

Muitos têm sido os trabalhos que visam estudar as relações entre fatores comportamentais - entenda-se por comportamentais, "comportamentos observáveis", "cognições" e "emoções" - e condições médicas - entenda por condições médicas todo tipo de transtorno físico.

Segundo o Manual Diagnóstico e Estatístico de Transtornos Mentais - DSM-IV (APA, 2000), a principal característica para o enquadramento na classificação de Fatores Psicológicos que Afetam a Condição Médica é a presença de fatores comportamentais que afetam adversamente uma condição médica. Estes fatores podem afetar a condição médica tanto no curso, como na precipitação ou no tratamento do quadro clínico. Para a Classificação de Transtornos Mentais e de Comportamento da CID 10 (WHO, 1998), esta mesma classificação recebe o nome de Fatores Psicológicos e de Comportamento Associados a Transtornos ou Doenças Classificados em Outros Locais. Segundo a WHO (1998) para validar esta classificação torna-se necessária a apresentação de uma condição médica.

Abaixo apresentar-se-ão alguns dos principais fatores comportamentais que têm sido estudados.

Um destes fatores é a ansiedade que, segundo Barros Neto (2000), é uma emoção que se assemelha a outra, que é o medo, tendo como diferencial entre elas a presença do estímulo no segundo.
Ansiedade, segundo Gentil (1997), é uma associação de sintomas somáticos - taquicardia, sudorese, dores, contraturas, sufocação, etc. - e psíquicos - tensão, insegurança, mal-estar, despersonalização, etc.

Segundo Calhoun e Resick (1999), a ansiedade parece estar ligada ao apoio familiar inadequado e à sensação de vulnerabilidade.

Outro fator que tem sido estudado com evidência e parece estar ligado a muitas condições médicas é a Depressão. Machado (1993) diz que transtornos psicológicos, como a depressão, estão intimamente ligados a algumas condições médicas.

Segundo o DSM-IV, a depressão é caracterizada por um estado debilitado do humor presente por um dado período de tempo. Para o modelo cognitivo-comportamental, ela tem como principal fator desencadeador a tríade cognitiva (visão de si, do mundo e do futuro) e é composta, principalmente, por: cognições, comportamentos e fatores bioquímicos (Beck, Rush, Shaw e Emery, 1997; Young, Beck e Weinberger, 1999) podendo, ainda, sofrer grande influência do ambiente, principalmente pela ausência de reforço (Ito e Lotufo-Neto, 2000).

Este transtorno pode prejudicar o desempenho do indivíduo não só profissionalmente, mas também suas relações interpessoais (Ito, Lotufo-Neto, Roso e Wielenska, 1998).

Diversos são os estudos (Gómez e Cols, 1989; Munoz e Cols, 1992; Malagris, 1996; 
Ayres, 1996; Alcino, 1996; Pinto, 1996) que visam observar relações entre condições comportamentais e algumas doenças tidas como multifatoriais. As doenças multifatoriais são aquelas que têm em sua etiologia influências de fatores diversos (Ayres, 1996). Algumas doenças tidas como multifatoriais são: hipertensão arterial, doença arterial coronariana, acidente vascular cerebral, esclerose múltipla, doença de Parkinson, epilepsia, síndrome do cólon irritável, enterite regional, colite ulcerativa, dispepsia, doença ulcerosa péptica, úlcera gastroduodenal, vitiligo, prurido, hiperidrose, dermatite atópica, urticária, rosácea, alopecia, psoríase, bronquite, asma, artrite reumatóide, diabete melito, entre outras.

Uma das formas mais comuns de avaliar condições comportamentais na população brasileira é através de instrumentos baseados em relatos verbais.

Hutz e Nunes (2001) criaram a Escala Fatorial de Ajustamento Emocional/Neuroticismo (EFN) que tem como finalidade avaliar a personalidade humana e baseia-se no relato verbal do sujeito. Este instrumento é composto por quatro sub-escalas (Ansiedade, Depressão, Vulnerabilidade e Desajustamento Psicossocial). A validação e normatização deste instrumento foram feitas com a participação de 1176 universitários de ambos os sexos e diferentes estados, os quais serviram para estabelecer escores bases para avaliações.

O presente estudo visou levantar fatores comportamentais, mais especificamente ansiedade e depressão, em uma amostra de pacientes classificados como portadores de fatores psicológicos que afetam as condições médicas e que tinham como condição médica associada uma das doenças consideradas multifatoriais, especificamente hipertensão, diabete melito, bronquite e asma.

\section{MÉTODO}

\section{Sujeitos}

Foram sujeitos do presente estudo: 30 pacientes que procuraram um Hospital Geral localizado na periferia da Zona Sul da cidade de São Paulo, de ambos os sexos e que foram classificados pelos médicos como portadores de Fatores Psicológicos que Afetam a Condição Médica.

Segundo a CID 10 (WHO, 1993), é necessário adicionar um transtorno físico para se classificar o indivíduo nesta condição. Os sujeitos desta pesquisa apresentavam transtornos físicos diversos, como: hipertensão, diabete melito, bronquite e asma.

\section{Material}

Foi utilizada, para a realização da pesquisa, a Escala Fatorial de Ajustamento Emocional/Neuroticismo (EFN). Esta consiste em 82 sentenças que descrevem atitudes, crenças e sentimentos e que foram pontuadas em uma escala tipo Likert, na qual "1" era assinalado quando a frase representava total inadequação com a característica do indivíduo, "7" quando representava total adequação e os números intermediários sendo equivalentes ao grau de concordância, tendo o "4" como neutro. Este instrumento foi criado e desenvolvido por Hutz e Nunes (2001).

\section{Procedimento}

O procedimento de coleta de dados foi realizado pelo pesquisador.

Os sujeitos que procuravam o hospital geral e eram classificados pelos médicos como portadores de Fatores psicológicos que afetam a condição médica e que tinham como condição médica associada uma das doenças conhecidas como multifatoriais, eram encaminhadas para a psicologia. Ao chegar lá o sujeito recebia esclarecimentos sobre a 
pesquisa e era convidado a participar da mesma, estando ciente de que a sua recusa não interferiria no seu atendimento junto ao hospital. Após concordância do paciente, através de carta de consentimento assinada, iniciava-se a aplicação do instrumento. Este procedimento se repetiu até que se completasse o total de sujeitos da amostra.

\section{RESULTADOS E DISCUSSÃO}

Após o procedimento de coleta, os sujeitos foram classificados segundo o posto percentil da normatização de Hutz e Nunes (2001), para posterior caracterização.

A Figura 1 traz os dados referentes às classificações da amostra, na sub-escala de ansiedade (N3) da EFN em comparação com a amostra de validação do instrumento. Os sujeitos foram classificados segundo o posto percentil da normatização de HUTZ e NUNES (2001). Os que tiveram pontuação igual ou abaixo de 46 (mulheres) e 41 (homens) são os que se encontram abaixo de $10 \%$ no posto percentil, e foram classificados como muito baixo. Os que tiveram pontuação entre 47 e 58

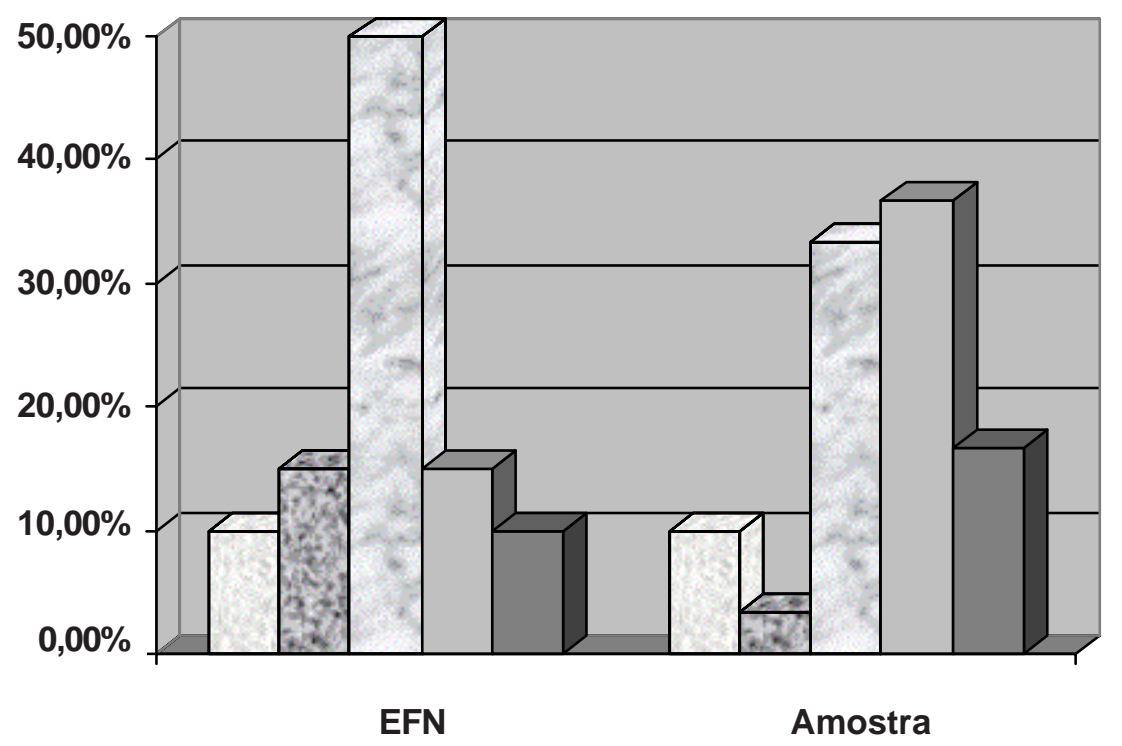

Muito abaixo da Média

Abaixo da Média

Média

Acima da Média

Muito acima da Média

Figura 1. Distribuição das freqüências percentuais da amostra estudada em comparação com a amostra de validação da sub-escala de Ansiedade da EFN. 
(mulheres) e 42 e 52 (homens) são os que se encontram entre $10 \%$ e $25 \%$ no posto percentil, e foram classificados como abaixo da média. Os que tiveram pontuação entre 59 e 92 (mulheres) e 53 e 85 (homens) são os que se encontram entre $25 \%$ e $75 \%$ no posto percentil, e foram classificados como média. Os que tiveram pontuação entre 93 e 109 (mulheres) e 86 e 100 (homens) são os que se encontram entre $75 \%$ e $90 \%$ no posto percentil, e foram classificados como acima da média. Os que tiveram pontuação igual ou acima de 110 (mulheres) e 101 (homens) são os que se encontram acima de $90 \%$ no posto percentil, e foram classificados como muito acima da média.

Encontrou-se na amostra portadora de doenças físicas, consideradas multifatoriais, uma maior concentração nas pontuações acima da média, se comparada com a amostra da EFN. Altos escores encontrados nas respostas são indicativos de desestabilidade emocional, com oscilações de disposição e humor, irritabilidade; também indicam sintomas como problemas no sono, impulsividade, podendo até chegar a fugas de pensamentos, que são sintomas ou sinais de ansiedade.

Estes achados, no entanto, devem ser vistos com cautela pois, apesar de reforçar a maioria das pesquisas da área, podem ter sofrido o efeito de variáveis intervenientes importantes. Por exemplo, é o caso da maioria das pesquisas que medem a ansiedade dos indivíduos, baseando-se em relatos verbais dos mesmos. Outro fator importante é que a literatura que estuda pacientes portadores de quadros multifatoriais não estuda, em sua maioria, pacientes com outras enfermidades com a função de controlar essas variáveis. Apesar de o presente estudo ter tomado como grupo controle a amostra de validação da EFN, esta foi composta por pessoas recrutadas em ambiente universitário e não em ambiente hospitalar.
$\mathrm{Na}$ Figura 2 encontram-se as classificações da amostra deste estudo, na sub-escala de depressão (N4) da EFN em comparação com a amostra de validação do instrumento. Os sujeitos foram classificados segundo o posto percentil da normatização de HUTZ e NUNES (2001). Os que tiveram pontuação igual ou abaixo de 25 (mulheres) e 26 (homens) são os que se encontram abaixo de $10 \%$ no posto percentil, e foram classificados como muito baixo. Os que tiveram pontuação entre 26 e 29 (mulheres) e 27 e 31 (homens) são os que se encontram entre $10 \%$ e $25 \%$ no posto percentil, e foram classificados como abaixo da média. Os que tiveram pontuação entre 30 e 49 (mulheres) e 32 e 50 (homens) são os que se encontram entre $25 \%$ e $75 \%$ no posto percentil, e foram classificados como média. Os que tiveram pontuação entre 50 e 63 (mulheres) e 51 e 68 (homens) são os que se encontram entre $75 \%$ e $90 \%$ no posto percentil, e foram classificados como acima da média. Os que tiveram pontuação igual ou acima de 64 (mulheres) e 69 (homens) são os que se encontram acima de $90 \%$ no posto percentil, e foram classificados como muito acima da média.

De acordo com a Figura 2, pode-se verificar que há uma maior concentração de sujeitos portadores de doenças físicas tidas como multifatoriais, com pontuações acima da média, na sub-escala de Depressão, se comparados com os indivíduos tomados como grupo controle (sujeitos que compuseram a amostra de validação e normatização da EFN).

Verificou-se que quatro em cada cinco sujeitos entrevistados, o equivalente a $80 \%$ da amostra total, encontram-se acima ou muito acima da média. Estes dados, segundo indicado por HUTZ e NUNES (2001), indicam que os sujeitos da amostra apresentam baixa expectativa quanto ao futuro, pouco ou nenhum objetivo a buscar, consideram-se solitários e possuidores de vidas monótonas, o que são indicativos de depressão. 


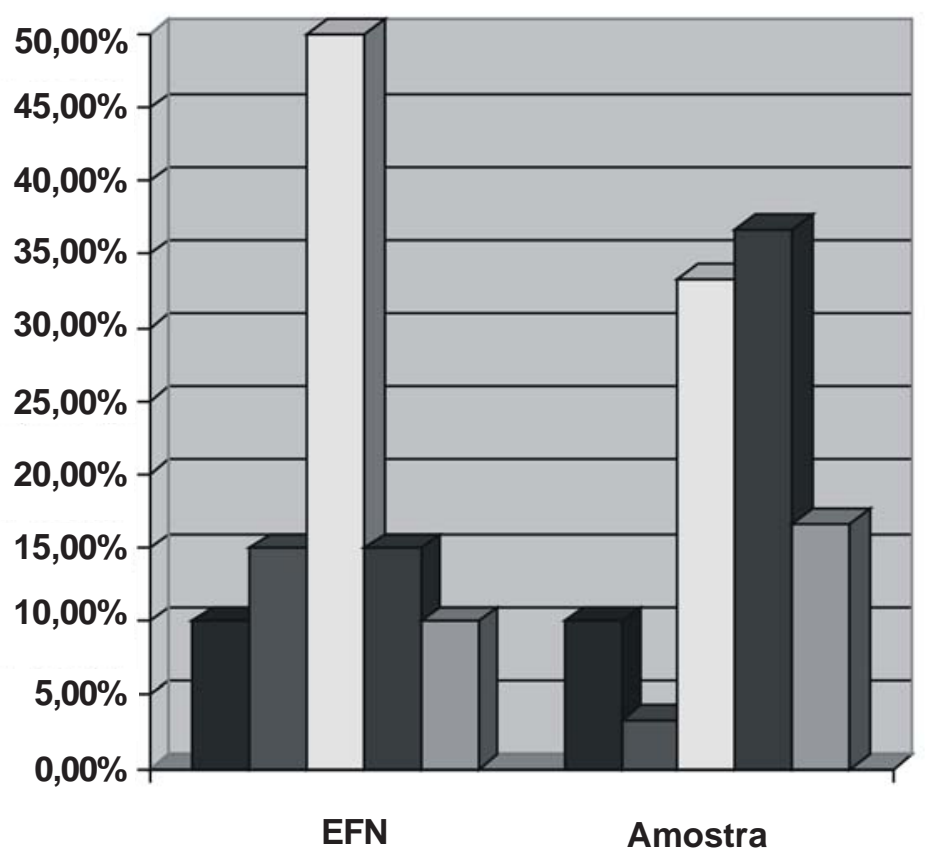

Muito abaixo da Média

$\square$ Abaixo da Média

$\square$ Média

$\square$ Acima da Média

$\square$ Muito acima da Média

Figura 2. Distribuição das freqüências percentuais da amostra estudada em comparação com a amostra de validação da sub-escala de Ansiedade da EFN.

No entanto, estes dados devem receber o mesmo cuidado mencionado na discussão da Figura 1, ou seja, não se controlaram algumas variáveis importantes, além de ter sido baseados em relatos verbais.

\section{CONCLUSÃO}

Verificou-se que o grupo de portadores de doenças físicas consideradas multifatoriais obteve altos escores de ansiedade e depressão na EFN, se comparado com o grupo de validação da mesma. Estes altos índices parecem reforçar a necessidade do trabalho do psicólogo junto a esta população.

Fica evidente que os níveis de ansiedade e depressão levantados na amostra da pesquisa encontram-se em maior concentração acima da média, o que pode levar a reflexões sobre a causa destas diferenças. É possível que estas diferenças sejam devidas ao fato de estarem doentes, pode ser porque são característicos da população com quadros multifatoriais, podem ser devidos ao nível socioeconômico diferenciado, entre outras 
hipóteses. Enfim, são muitas as variáveis que necessitam ser controladas nestes estudos a fim de se conseguir resultados mais confiáveis sobre a existência ou não de relações entre ansiedade e depressão e doenças multifatoriais. Se existirem tais relações, em quais situações ocorrem, como e porque.

São necessários novos estudos visando confirmar estes dados. No entanto, sugere-se que estes tenham como grupo controle pacientes que estejam portando enfermidades não consideradas multifatoriais para que se possa estudar se a depressão e a ansiedade são características de todos os doentes, portadores de condições multifatoriais ou não. Outra sugestão é que estes estudos se utilizem de métodos diferentes de relatos dos sujeitos, pois assim poder-se-á eliminar esta variável, que pode ser interveniente.

\section{REFERÊNCIAS BIBLIOGRÁFICAS}

ALCINO, A. B. (1996). Stress social e reatividade cardiovascular infantil: um estudo psicofisiológico. Em M. E. N. Lipp (Org.). Pesquisas sobre stress no Brasil: Saúde, ocupação e grupos de risco (pp. 35 -46). Campinas: Papirus.

AMERICAN Psychiatric Association (2000). Manual Diagnóstico e Estatístico de Transtornos Mentais - DSM-IV, $4^{a}$ ed. (Trad. D. Batista). Porto Alegre: Artes Médicas Sul. (Originalmente publicado em 1994).

AYRES, A. M. M. (1996). Stress e afetividade nos hipertensos. Em M. E. N. Lipp (Org.). Pesquisas sobre stress no Brasil: Saúde, ocupação e grupos de risco (pp. 71-81). Campinas: Papirus.

BARROS NETO, T. P. (2000). Sem Medo de Ter Medo: um guia prático para ajudar pessoas com pânico, fobias, obsessões, compulsões e estresse. São Paulo: Casa do Psicólogo.

BECK, A. T. Rush, A. J. Shaw, B. F e Emery, G. (1997). Terapia Cognitiva da Depressão
(Trad. S. Costa) Porto Alegre: Artes Médicas. (Originalmente publicado em 1979).

CALHOUN, K. S. e Resick, P. A. (1999). Transtorno do estresse pós-traumático. Em D. H. Barlow (Org.). Manual Clínico dos Transtornos Psicológicos $2^{a}$ ed (pp. 63 - 118). Porto Alegre: Artmed. (Originalmente publicado em 1993).

GENTIL, V. (1997). Ansiedade e transtornos de ansiedade. Em V. Gentil, F. Lotufo-Neto e M. A. Bernik (Org's.). Pânico, Fobias e Obsessões: a experiência do Projeto AMBAN (pp. 29 - 36). São Paulo: EDUSP.

GOMEZ, L. De Las N. A Romero, N. M. Machin, J. V. M; Zuñiga, M. B. e Ruiz, A. G. (1989). Asma bronquial, estres y síndrome psicofisiológico dominante. Revista Del Hospital Psiquiátrico de la Habana, 30, (4), 551-558.

HUTZ, C. S. e Nunes, C. H. S. S. (2001). Escala Fatorial de Ajustamento Emocional/ Neuroticismo - EFN. São Paulo: Casa do Psicólogo.

ITO, L. M. e Lotufo Neto, F. (2000). Teorias cognitivo-comportamentais, interpessoal e construtivista. Em B. Lafer, O. P. Almeida, R. Fraguás Jr. e E. C. Miguel, (Org's.). Depressão no Ciclo da Vida (pp. 82 - 91). Porto Alegre: Artes Médicas Sul.

ITO, L. M. e Lotufo Neto, F. Roso, M. C. e Wielenska, R. (1998). Depressão. Em L. M. Ito (Org.). Terapia Cognitivo-comportamental para Transtornos Psiquiátricos (pp. 91 - 103). Porto Alegre: Artes Médicas.

MACHADO, A. G. (1993). La somatización y los transtornos somatomorfos en la práctica médica. Gaceta Médica de Caracas, 101, (2), 101-106.

MALAGRIS, L. E. N. (1996). Stress e úlceras gastroduodenais: interações clínico-psicológicas. Em: M. E. N. Lipp (Org.). Pesquisas sobre stress no Brasil: Saúde, ocupação e grupos de risco (pp. 83-108). Campinas: Papirus. 
MUNOZ, R. A; Salazar, D. A Alfaro, J; Vera, A; Toledo, M. I. Serrano, F; Valdivia, C. G; Nunez, C; Godoy, S. e Soto, O. (1992). Comparación de variables psico-sociales entre un grupo con transtornos emocionales y uno control. Revista de Psiquiatria, 9, (2), 1141-1152.

PINTO, W. N. R. (1996). Stress, qualidade de vida e vitiligo. Em M. E. N. Lipp (Org.). Pesquisas sobre stress no Brasil: Saúde, ocupação e grupos de risco (pp. 129-148). Campinas: Papirus.
YOUNG, J. E. Beck, A. T. e Weinberger, A. (1999). Depressão. Em D. H. Barlow (Org.). Manual Clínico dos Transtornos Psicológicos $2^{a}$ ed. (pp. 273-312). Porto Alegre: Artmed. (Originalmente publicado em 1993).

WORLD Health Organization (1998). Classificação de Transtornos Mentais e de Comportamento da CID 10 (Trad. M. L. Domingues). Porto Alegre: Artes Médicas. (Originalmente publicado em 1993). 